ON

\title{
THE LIGAMENTS
}

or 2xis

\section{HUMAN OSSICULA AUDITUS.}

Br THOMAS WILLIAM CHEVALIER, Esq.

Read April 27, 1824.

IT is generally allowed at the present day, that all new facts, however apparently fruitless when recently discovered, should be recorded for the consideration of the many who are devoting their time and their talents to philosophical pursuits. And indeed it is only by those generous principles of conduct, whereby the mind of the world has been, at the earliest period, employed upon the observations of individuals, that we can possibly account for the recent advancement of science.

To a medical student of the present day, even the period of his own pupilage presents instances in which the progress of Pathology appears to have been the immediate result of perseverance in anatomical research; while the diseases which most resist the skill, and baffle the reasoning of the nosologist,-for example, those of the nerves, of the skin, of the ear, \&rc.-are precisely those concern- 
ing which our anatomical knowledge has been hitherto most glaringly deficient.

My attention having been accidentally directed about six or seven years ago, to a minute investigation of the human organ of hearing, and having been unable to quit so interesting a subject, I began to consider whether the ossicula auditus might not, like other bones of which an accurate movement is required, be restrained and directed by a set of ligaments.

Though joints so minute must have very small ligaments, if any, I hoped that the accurate knowledge of them, might at least enable me to form a decided opinion upon the question, whether the mechanism contained in the cavity of the tympanum, adapts itself to the force alone, or also to the specific and individual characteristics of those impulses which it transmits.

The columella in the ears of birds is weakest in those that sing best. It bends under almost the slightest discernible impulse that can be communicated to the membrana tympani of these animals. If, for example, the membrana tympani of the thrush, or of the nightingale, were to receive the rude impulse of a touch, such a force would be immediately impressed upon the contents of the labyrinth as to injure them, were it not for the extreme flexibility of the columella. So that the pre- 
servation of the delicate auditory nerve of these birds appears due to the weakness of the recipient or conductor of all they hear; and this defensive provision appears one important use of the sole ossiculum, - or rather of the sole cartilago auditus, that they possess. But one other purpose is evidently answered by the columella in the ears of birds, viz. the diminution of the area of the membrana tympani to that of the fenestra vestibuli.

In man, the incus does not communicate immediately with the stapes, but, as every one knows, through a double ball-and-socket joint; and the stapedius muscle is so placed, that it cannot fail, as often as it acts, to draw the latter bone from its usual position, under the arm of the incus; and to relieve the contents of the labyrinth from the direct impression of too violent a shock (Fig. 2. No. 12).

It is proper to remark, that very few of the ligaments of the body are so precisely similar, with respect to their forms and positions in different subjects, as to answer accurately the descriptions of Weitbretch ; although his work upon those parts is the admiration of all who have once employed it in dissection, and can never be excelled. Accordingly, the ligaments of the ossicula auditus (which I have described in the explanation of the drawings as new to me) have not always precisely the same position or form. Several are of a quadrangular shape, but they have their angles in different indi- 
viduals more or less acute. I have found the ligaments A, D, E, F, in some instances nearly parallel, in others, nearly perpendicular to the horizon. These four, however, as well as $\mathbf{G}$ and $\mathbf{H}$ are always so placed as to accomplish one uniform object, viz. the restriction of the-movement of the head of the malleus, and therefore, also that of its manubrium, to one direction: and again, they are always so placed as to be put on the stretch by the elasticity of the drum of the ear; hence their effect, to prevent the membrana tympani from becoming less curved, and to oppose its relaxation, excepting when that purpose is accomplished by the musculus laxator tympani major.

To discover the ligaments of the human ossicula auditus, it is necessary to bear in mind that they are in most subjects extremely delicate, nearly transparent, and easily torn up with the periosteum lining the cavity of the tympanum. In making the preparations from which the accompanying drawings are taken * the cells of the petrous portion of the temporal bone were sawn away, an opening was thus made into the cavity of the tympanum, at the exterior and posterior part of its roof. The edges of this opening I carefully cut and pared away with the point of a knifet, carefully observ-

* These preparations with some others still to be made, will be deposited in the Hunterian Museum of the Royal College of Surgeons.

+ The knife that I used has a large strong handle, into which 
ing that my instrument should not remove even a point of membrane, or of bone, more than $I$ intended, or more than I could accurately and certainly watch. Proceeding in this cautious manner there appears under the edge of the opening, the head of the malleus; or perhaps first, a very small but comparatively substantial ligament (B) uniting the head of the malleus to the roof of the tympanum. I have sometimes thought this was a capsular ligament; I cannot, however, satisfy 'myself that it contains any cavity. Manifestly it restricts the mobility of the head of the ossiculum, in all directions, to a certain extent; and may steady it also, both directly, and by obviating any vibration of its parts. Avoiding this suspensory ligament (for such it is in position, though not in office), and sparing the points of bone to which it is attached, the rest of the roof of the tympanum may be cut away; and there will thus be exposed a set of ligaments (A, D, E, F) together with the triangular body of the incus, completing, at the least four-sevenths of a circle, around the exterior of the neck of the malleus.

Now, taking care not to dislocate the stapes, the fenestra ovalis and all the interior wall of the

was screwed a short pointed blade of hard steel, with its sides meeting from a thick back, at a considerable angle, in the edge. With a single blade, for which the author is indebted to Mr. Stodart, he dissected six petrous bones, before the point was broken. His saw was a watch-spring saw.

VOL. XIII. 
tympanum may be with care and perseverance removed : and thus, in addition to that semicircular restraint upon the malleus already mentioned, as leaving it moveable in no other direction than outwards, there will be found two more ligaments ( $G$ and HK), whereof one is twofold.

Very commonly the short process of the malleus, at the obtuse angle between the manubrium and neck of this ossiculum, is as it were hitched upon the bony ring surrounding the membrana tympani : and hence, and more especially because at the same part of the malleus the long process goes off like an axle-tree, this appears to be the fulcrum upon which the malleus, as a lever, is moved.

In some instances, however (as in the sketch fig. 3.), this fulcrum or angle of the malleus is very far from being supported under its movements upon the auditory process or ring: the ossiculum being on the contrary suspended entirely within the circle of the membrane.

In either case, the angle of the external ossiculum, and for a short distance its manubrium, are supported by two very firm ligaments $G$ and $H$ : both of these ligaments being in a plane which is at an acute angle with that of the upper margin of the membrana tympani: both of these ligaments being so situated as to correspond in the restraint 
they lay upon the malleus, with that of the ligaments around the exterior of its neck.

From the edge of that ligament which is posterior to the manubrium mallei, viz. from the edge of the ligament $\mathrm{H}$, there proceeds, at an angle with: its plane, a ligamentous band which is attached along the side of the articulating or long process of the incus: and sometimes fibres of this ligamentous band pass in a curve immediately from the manubrium mallei to the processus longus incudis. So that, whereas the ligaments $A, D, E$, $F, G, H$, confine the movement of the malleus, and therefore that of its manubrium to one single direction, the ligamentous band $K$ prescribes this very same general direction, and probably the same extent, to the movement of the articulating or long process of the incus: that is, in effect, to the stapes itself. Also I have seen, in an ear of which the sense was unimpaired, the whole length of the articulating process of the incus, and the whole of the stapes, bound down to the interior wall of the cavity of the tympanum; viz. to that which is opposite to the membrana tympani.

As to the effects of the ligaments $A, B, C, D$, $E, F, G, H$, and $K$, considered in reference to the actions of the muscles of the malleus; the ligamentous attachments last described obviously suggest, in the first place, that the malleus and incus do not act as two distinct levers upon each other, 
increasing the strength or extent of those sonorous vibrations which the drum of the ear receives. And, indeed, when we attentively examine the firm grasp of the incus upon the head of the malleus, and find the capsular ligament between these bones invariably the tightest in the body, it is difficult, if not impossible, to believe that these two ossicula act independently, or in such a way as must vary the tension of those ligamentous fibres that are found to unite, to no inconsiderable extent, their most moveable processes or arms (fig. 4. $\mathrm{K}^{*}$ ).

From the extremity of the short process, at the angle of the malleus, where it protrudes immediately below the uppermost part of the tube, or ring on which the membrana tympani is stretched; there proceeds a little pencil of fibres, which, diverging upwards, is inserted into the auditory process, or ring, immediately above. To this conical pencil of fibres Albinus restricts the name, laxator tympani ; and if these fibres be muscular, they must tend to relax the membrana tympani, by reducing its curved surface nearer to a plane. Such an effect however will evidently be opposed by all the ligaments of the ossicula auditus: and hence this very short and conical muscle,-if such it be, has

* A patient of my father's, as also several others whose cases are upon record, could still hear with an ear from which he had lost all the ossicula auditus: $-\boldsymbol{A}$ fact which is scarcely credible if the little bones really tripled or quadrupled, as has been supposed, the extent of sonorous impulses or vibrations. 
the remarkable office of steadying the malleus; or perhaps even that of holding it at rest, by restraining the only movement permitted by its ligaments.

The musculus externus mallei of Albinus, anterior mallei of Winslow, or laxator tympani of most anatomists is always, as far as I have observed, composed of very straightened and distinct fasciculi, and so tendinous and pale in its appearance as frequently to resemble rather a ligament than a muscle. It is known to be inserted into the malleus by the long process of this ossiculum, and so that the process might be considered as an osseous tendon belonging to the muscle in question.

If this muscle be pulled in the dead bone, the membrana tympani of the infant is indeed relaxed; but the malleus is not moved upon its fulcrum, for the osseous tendon of the muscle is in effect as an axle-tree* to the ossiculum or lever. In the action mentioned the whole malleus is evidently moved in a direction downwards, forwards, and a little outwards; and this change of the place of the whole ossiculum in this direction, is not opposed by any of the ligaments. For example, the ligament $\mathrm{H}$-though it would oppose the movement or displacement of the malleus forwards, is to be somewhat relaxed in a direction

* This expression, although inaccurate, appeared to me that which is most likely to be understood. (See figs. 3. and 4. No. 15.) 
outwardsand downwards, and it is therefore steadily moveable in the mean direction forwards, outwards, and downwards. And so of the ligaments $A, B$, D, E, F, or G. Clearly enough, the elasticity of the membrana tympani, is sufficient to relax it as far as it can be relaxed without being thrown into folds; that is, if unopposed : and when the musculus externus mallei, as it is called, is pulled in the infant's bone, the membrana tympani is thrown into folds, and rendered (as one would suppose) wholly incapable, for the time, of sonorous vibration. In general, as muscles are required to act for longer periods at once, they are more tendinous. During sleep not only are the eye-lids closed, but the cornea is elevated, as observed by Mr. Bell, in the Phil. Trans. for 1823, and sustained behind the upper palpebra by the inferior oblique muscle of the eye. Has then the musculus externus mallei an analogous use, diminishing the susceptibility of the ear for sounds during sleep? or does this muscle serve only to hold firm the malleus (as in the bones of most adults) against the extremity of that tubular interstice in which is lodged the osseous tendon of the musculus externus mallei ? In many bones I have been able but thus to balance, as it were, the malleus, and to hold it steady against the extremity of the interstice when $I$ have pulled the muscle; and by these means I have seemed to render it more apt to receive the sonorous vibration : there being no space left between the interstice and the malleus, and therefore no vi- 
sible displacement of this ossiculum possible. (Figs. 3, 4. No. 15.) The tensor tympani is a considerable fleshy muscle, as large as a ripe oat; it draws the manubrium of the malleus directly inwards, relaxes every one of the ligaments which restrain this ossiculum, and allows its head, therefore, a 'slight primary movement inwards; at the same time it presses all the articulating surfaces of the ossicula into contact, each with its natural correspondent, and thus prepares the membrana tympani and the ossicula auditus for the reception and transmission of the most delicate sounds to the auditory nerve.

The tendon of this interesting muscle is, however, so closely united at the point where it is bent to a right angle, to the interior wall of the cavity of the tympanum, that it is prevented from moving to an injurious extent.

When the tensor tympani is in action, and the membrana tympani stretched inwards, we can easily imagine that it might be rendered yet more vibratory and elastic, with perfect safety, could it be endowed with a variable power, within itself, of opposing its own extension inwards, according to circumstances. And this is the fact; for physiology is indebted to the talents and research of Sir Everard Home, who has accurately, and, I think, satisfactorily proved that the membrana tympani is muscular. 
72 ON THE LIGAMENTS OF THE OSSICULA AUDITUS.

With respect to the labyrinth of the human ear, I have made several observations, which I believe are not generally known, but which I wish to reconsider, from the difficulty I have found of late in obtaining a sufficient number of recent bones to dissect.

South Audley Street, April 27th, 1824. 


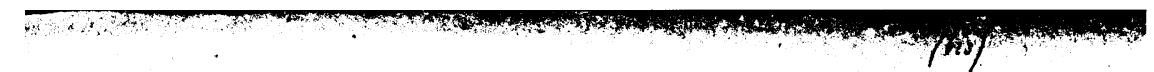

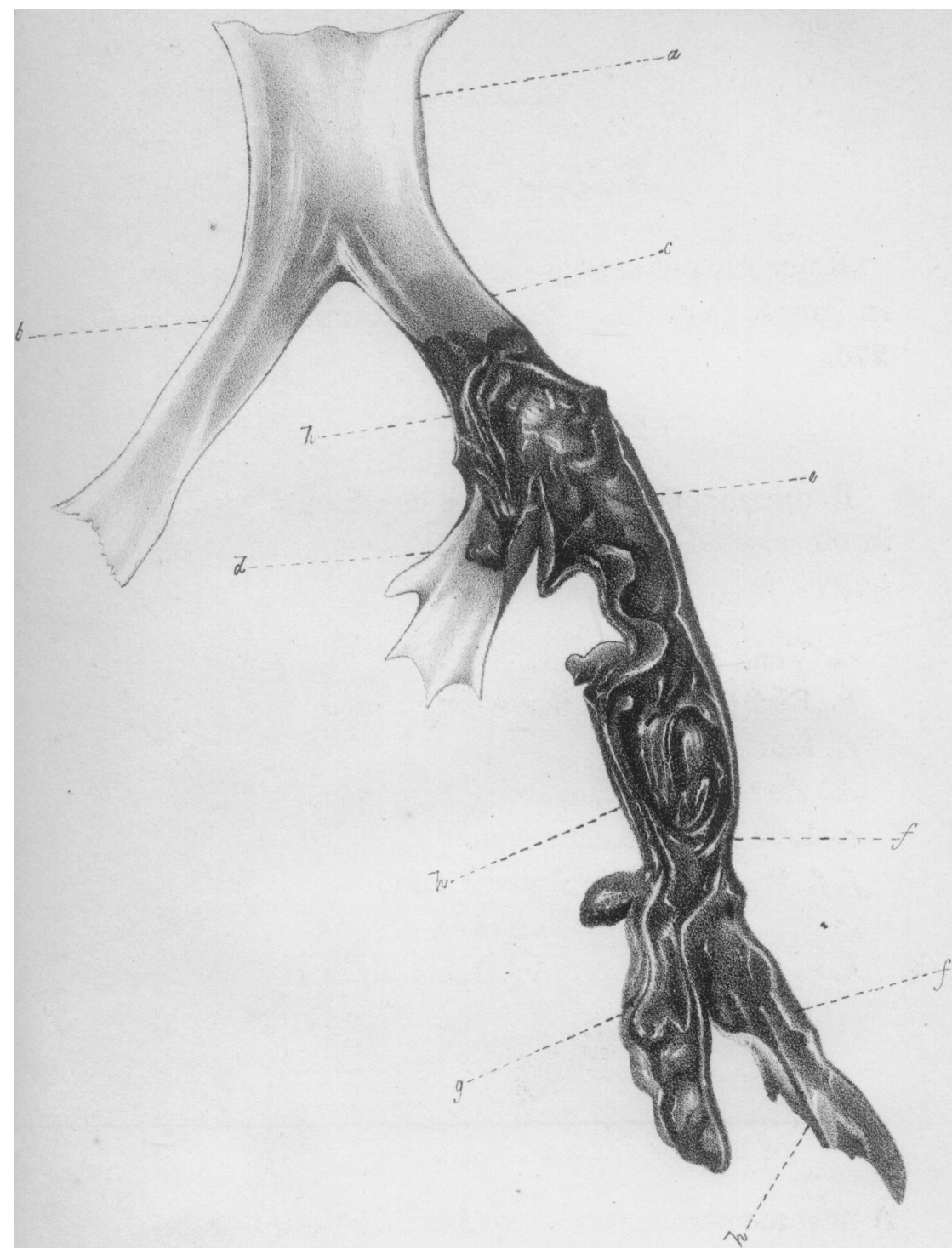

